\title{
A Variable-Fidelity Modeling Method for Aero-Loads Prediction
}

\author{
Z.-H. HAN, S. GÖRTZ and R. HAIN \\ Deutsches Zentrum für Luft- und Raumfahrt e.V., \\ Institut für Aerodynamik und Strömungstechnik, \\ Lilienthalplatz 7, 38108, Braunschweig, Germany \\ Zhong-hua.Han@dlr.de, Stefan.Goertz@dlr.de
}

\section{Summary}

A Variable-Fidelity Modeling (VFM) method has been developed as an efficient and accurate aerodynamic data modeling strategy. In this approach, a set of CFD methods with varying degrees of fidelity and computational expense is exercised to reduce the number of expensive high-fidelity computations. Kriging-based bridge functions are constructed to match the low- and high fidelity CFD data. The method is demonstrated by constructing a global approximation model of the aerodynamic coefficients of an RAE 2822 airfoil based on sampled data. The model is adaptively refined by inserting additional samples. It is shown that the method is promising for efficiently generating accurate aerodynamic models that can be used for the rapid prediction of aerodynamic data across the flight envelope.

\section{Introduction}

From an aerodynamic point of view, an aircraft is defined by comprehensive datasets regarding performance, loads and handling characteristics. This data, which needs to be determined for every possible flight condition, is used to design the structure of the aircraft and the flight control system. Currently, this data is obtained mainly from costly wind tunnel tests or using hand-book methods. The use of higher-fidelity and thus more time consuming CFD methods has been, up to now, impossible due to the large volume of data required. The long-term goal of the work described here is the development of a process chain for the efficient numerical prediction of all relevant aerodynamic data for the elastic aircraft over the entire flight envelope, based on a hierarchy of CFD methods of increasing fidelity. The idea is to use Variable-Fidelity Modeling (VFM).

The idea of VFM is not entirely new since it has been used in the aircraft design optimization community for more than ten years. Examples of local VFM, where an approximation model is constructed using only a few data points nearest to the design point of interest and refined during optimization, can be found in Ref. [1-4]. Examples of global VFM, where the approximation model is constructed with all the available points throughout the parameter space, can be found in Ref. [5-6]. Very few publications on VFM in the context of aerodynamic data for loads are available. In Ref. [7], e.g., a so called "data fusion" method was developed for the generation of an aerodynamic database by using CFD tools of varying fidelity.

This paper focuses on the development a VFM method that is especially well suited for predicting and modeling the aerodynamic data of aircraft throughout the 
entire flight envelope. Kriging-based global approximation of the aerodynamic data and Kriging-based bridge functions are studied and used to construct a global surrogate model of the aerodynamic data in the parameter space. A novel samplepoint refinement strategy is proposed to adaptively refine the model, which makes it more accurate and the VFM method more efficient. The developed method is demonstrated for the prediction of the aerodynamic coefficients and the drag polar of an RAE 2822 airfoil at different Mach numbers and angles of attack.

\section{Framework of the Variable-Fidelity Modeling Method}

The framework for VFM was designed for constructing a model that can approximate the high-fidelity data throughout the parameter space. It is depicted in Figure 1. The basic steps of this framework are as follows:

- Step 1 Initialization: Define the unknown aero-loads (integrated or distributed) to be modeled; specify the parameter space by defining the independent variables and their range.

- Step 2 Sampling: Two sets of sample points (called samples in the following) are generated based on Design of Experiment (DoE) theory; one is for the intensively samples parameter space to be computed with the low-fidelity method, the other is for the less intensively samples parameter space to be evaluated with the high-fidelity method.

- Step 3 Sample point evaluation: The aerodynamic data at the samples are calculated by low- and high-fidelity CFD methods, respectively.

- Step 4 Low-fidelity surrogate model and bridge function: Based on the lowfidelity sampled data, a Kriging model is constructed as a surrogate model to the low-fidelity CFD method (called low-fidelity Kriging). Based on the difference between the low-fidelity surrogate model and the high-fidelity data at the locations of the high-fidelity samples, a Kriging-based bridge function is constructed to match the low- and high-fidelity CFD methods.

- Step 5 Initial VFM construction: The low-fidelity surrogate model is corrected with the bridge function and an initial VFM is constructed.

- Step 6 Refinement: iterative refinement is performed by adding additional samples until a criterion for termination is fulfilled.

- Step 7 Final VFM for aero-loads prediction: Based on the final VFM, the parameter space can be probed in "real-time" for aerodynamic data at any point in the parameter space within the limits prescribed in Step 1 or a database of aerodynamic data can be efficiently generated by filling in the remainder of the parameter space using the VFM.

\section{Kriging Model and Bridge Function}

One of the key issues for VFM is how to create the global surrogate models. The Kriging method [9], a geostatistical technique, was used here due to its good global approximation characteristics. For a problem with $n_{v}$ variables and $n_{s}$ samples, the Kriging method approximates the unknown function $y$ by a linear combination of the known values at samples as follows 


$$
\hat{y}\left(\mathbf{x}_{p}\right)=\sum_{i=1}^{n_{s}} w_{i}\left(\mathbf{x}_{p}\right) y\left(\mathbf{x}_{i}\right), \mathbf{x} \in R^{n_{v}}
$$

where $\hat{y}\left(\mathbf{x}_{p}\right)$ is the predicted value at the location denoted by $\mathbf{x}_{p}, \mathbf{x}$ is vector of independent variable and $w_{i}$ are the weight functions. For the ordinary Kriging used in this study, the weight functions fulfill the unbiasedness condition

$$
\sum_{i=1}^{n_{s}} w_{i}\left(\mathbf{x}_{p}\right)=1
$$

By minimizing the mean squared error of the predictor with the above equivalent constraint, the ordinary Kriging predictor can be expressed as

$$
\hat{y}\left(\mathbf{x}_{p}\right)=\left[\begin{array}{c}
y_{1} \\
\vdots \\
y_{n_{s}} \\
0
\end{array}\right]^{T}\left[\begin{array}{cccc}
R\left(\mathbf{x}_{1}, \mathbf{x}_{1}\right) & \cdots & R\left(\mathbf{x}_{1}, \mathbf{x}_{n_{s}}\right) & 1 \\
\vdots & \ddots & \vdots & \vdots \\
R\left(\mathbf{x}_{n_{s}}, \mathbf{x}_{1}\right) & \cdots & R\left(\mathbf{x}_{n_{s}}, \mathbf{x}_{n_{s}}\right) & 1 \\
1 & \cdots & 1 & 0
\end{array}\right]^{-1}\left[\begin{array}{c}
R\left(\mathbf{x}_{1}, \mathbf{x}_{p}\right) \\
\vdots \\
R\left(\mathbf{x}_{n_{s}}, \mathbf{x}_{p}\right) \\
1
\end{array}\right]
$$

where $y_{1}, \ldots, y_{n}$ are the function values at samples; $R\left(x_{i}, x_{j}\right)$ is the spatial correlation function [9] between any two points $\left(\mathbf{x}_{i}\right.$ and $\left.\mathbf{x}_{j}\right)$.

Another key issue for VFM is how to manage the different models of varying fidelity or how to correct the low-fidelity surrogate to approximate the highfidelity data by making use of so called "bridge functions", which are sometimes called "scaling functions". The existing bridge functions can be divided into three categories: multiplicative [3], additive [5, 8] and hybrid [7]. All three approaches require the construction of an unknown function to correct the lower-fidelity model, which in turn will approximate the high-fidelity model. Note that the bridge functions can take the form of first- or second-order polynomials or Kriging models. To avoid the possible problem of diving by zero when using multiplicative bride functions, an additive bridge function was used in this study. The additive bridge function can be expressed as

$$
\gamma(\mathbf{x})=y_{h f}(\mathbf{x})-y_{l f}(\mathbf{x})
$$

where $y_{l f}(\mathbf{x})$ and $y_{h f}(\mathbf{x})$ denote the low- and high-fidelity models, respectively. After an additive bridge function $\gamma(\mathbf{x})$ is approximated by Kriging, the highfidelity model can be approximated by the following VFM:

$$
\hat{y}_{V F M}(\mathbf{x})=\hat{y}_{l f}(\mathbf{x})+\hat{\gamma}(\mathbf{x})
$$

\section{Sample-Point Refinement Strategy}

A refinement strategy dedicated to VFM has been developed. The method assumes that the VFM is always more accurate than the Kriging model constructed directly from the high-fidelity samples (called high-fidelity Kriging). Only in the limit of a very large number of high-fidelity samples, the VFM and the high-fidelity Kriging model will converge to the same result. Hence, one can measure the error between the VFM and the high-fidelity Kriging model to avoid the difficulty of having to estimate the error between the VFM and the exact underlying functional relationship, which is unknown. The error is defined as

$$
e_{i}=\left|y_{V F M}^{i}-y_{h}^{i}\right|, i=1,2, \ldots, n_{t}
$$


where $n_{t}$ is the number of test points, $y_{V F M}^{i}$ and $y_{h}^{i}$ denote the VFM and highfidelity Kriging value at the $i^{\text {th }}$ test point, respectively. The maximum, average error in \% and the RMS error are defined as

$$
e_{\max }=\max \left(e_{i}\right), \bar{e}=\frac{1}{n_{t}} \sum_{i=1}^{n_{t}}\left(\frac{e_{i} \times 100}{y_{V F M}^{i}}\right), R M S=\sqrt{\left(\sum_{i=1}^{n_{t}} e_{i}^{2}\right) / n}
$$

Since all the values used in the error estimation are obtained from the surrogate models, the computational cost is negligible even for a large number of test points. The location with the maximum error is selected for refinement, and the VFM is reconstructed. The refinement is repeated until the average error is below a certain threshold, here $5 \%$. This refinement method will be demonstrated and evaluated in the next section.

\section{Results and Discussion}

The VFM method is demonstrated by modeling the aerodynamic coefficients of an RAE 2822 airfoil as a function of one and two free stream flow parameters. The VFM framework was built around the DLR TAU code [10]. The low- and high-fidelity computations were performed with the Euler and RANS versions of the TAU code, respectively. Jameson's central scheme was used for space discretization, and an LUSGS implicit scheme was used for time stepping. The Spalart-Allmaras one-equation model was utilized for the simulation of fully turbulent flow. The grids around the RAE 2822 airfoil used for the Euler and RANS computations are illustrated in Figure 2.

A VFM framework based on a single physical model (TAU Euler) evaluated on meshes of varying refinement was also demonstrated for the RAE 2822 airfoil (not shown here). It was shown that in this case VFM works very well even without sample-point refinement as the low-fidelity model captures the overall trend of the high-fidelity model.

\subsection{VFM for RAE 2822 with One Independent Variable}

At first, the VFM was applied to model the aerodynamics of the RAE 2822 airfoil as a function of a single variable, angle of attack, $\alpha$, in the range from $-4^{\circ}$ to $16.5^{\circ}$. The Mach number was fixed at 0.2 . A Reynolds number of $6.5 \times 10^{6}$ was assumed for the RANS computations. 26 samples were selected for the Euler computations, whereas only 4 initial samples were selected for the RANS computations. The RANS computations were also performed at the locations of the 26 low-fidelity samples to validate the constructed VFM. The VFM was used to model the lift, drag and moment coefficients, as well as the drag polar. The VFMs for the lift and the drag polar are shown Figure 3. Reasonably good agreement between the VFM (without refinement) and the validation data is observed. Figure 4 shows the VFM for the moment coefficient as a function of angle of attack. The key features of VFM are clearly shown here: at low angles of attack the VFM is in very good agreement with the validation data, since the Euler method can capture the trend of the RANS method. At high angles of attack (especially near stall), the Euler method misses the trend, and the VFM is no longer better (but also not worse) than the high-fidelity Kriging model directly constructed from the high-fidelity samples. In such a case, refinement 
becomes necessary. The initial VFM was refined by adding new samples adaptively until the criterion for termination was fulfilled (see section 4). Five new samples were added to refine the VFM, mainly in the region in which the flow is dominated by nonlinear effects. The refined VFM is in very good agreement with the validation data. As one of the results of refinement, the accuracy of high-fidelity Kriging was also increased. Although the final VFM is only slightly better than the high-fidelity Kriging model, VFM can be used to adaptively refine the approximation model.

\subsection{VFM for RAE 2822 with Two Independent Variables}

The VFM was then demonstrated for a problem with two variables (or a twodimensional parameter space), angle of attack, $\alpha$, and Mach number, $M a$. The range of the variables was specified with $M a$ ranging from 0.1 to 1.2 and $\alpha$ ranging from $4^{\circ}$ to $10^{\circ}$. 345 samples were selected manually for the Euler computations, while 32 initial samples were specified for the RANS computations using a Quasi-Monte Carlo DoE method. Refinement had to be performed since the Euler method missed the trend in the transonic regime and at high angles of attack. Based on the VFMs for the lift, drag and moment coefficients, respectively, 3 new samples were specified at each refinement iteration step. 15 steps of refinement were performed, and a total of 44 new samples were added. All the samples are plotted in Figure 5. It is clear that most of the samples were added in the region in which the flow is dominated by nonlinear effects. The remaining samples were added at the border of the parameter space. The 3-D hypersurfaces of the drag coefficient versus Mach number and angle of attack are shown in Figure 6. The low-fidelity Kriging model constructed from the 345 Euler computations is shown in Figure 6a; the high-fidelity Kriging model constructed from the 32 RANS computations is shown in Figure 6b. The difference between the lowfidelity Kriging model and the high-fidelity samples was used to construct the Krigingbased bridge function, and the VFM was obtained by correcting the low-fidelity Kriging model with the additive bridge function. The comparison of the final VFM and the validation data is also shown in Figure 6c, and very good agreement is observed. By comparing the number of high fidelity samples needed to construct an accurate VFM and that need for validation, one can conclude that the number of RANS computations can be reduced by a factor of about 5 .

\section{Conclusions}

An efficient and accurate Variable-Fidelity Modeling (VFM) method for aeroloads prediction has been developed. This method was demonstrated for the prediction of the aerodynamic coefficients of an RAE 2822 airfoil. Some preliminary conclusions can be made as follows:

- When the low-fidelity model misses the actual trend in a specific region of the parameter space, the VFM needs to be refined in this region.

- The proposed refinement strategy was proven to be efficient and robust. It can be used to adaptively refine the VFM.

- The VFM method offers the possibility to significantly reduce the computational cost in the aero-data for loads context. 
To further improve the efficiency and accuracy of VFM, the so-called gradientenhanced direct Cokriging method will be employed. Also, the range of fidelity will be extended to include panel methods and RANS with wall functions. The method will also be extended to distributed aero loads and will be applied to more complex configurations and across the entire flight envelope.

\section{References}

[1] V. Braibant, C. Fleury. "An Approximation-Concepts Approach to Shape Optimal Design". Computer Methods in Applied Mechanics and Engineering, Volume 53, 1985, pp. 119-148.

[2] R.T. Haftka. "Combining global and local approximations". AIAA Journal, Volume 29, No.9,1991, pp. 1523-1525

[3] K.J. Chang, R.T. Haftka, G.L Giles, P.-J. Kao. "Sensitivity-based scaling for approximating structural response". Journal of Aircraft, Volume 30. No.2, 1993, pp.283-288.

[4] N.M. Alexandrov, R.M. Lewis, C.R. Gumbert, L.L. Green, P.A. Newman. "Optimization with variable-fidelity models applied to wing design". AIAA Paper 2000-0841, Jan. 2000.

[5] S. Cho, J.J. Alonso, I.M. Kroo, M. Wintzer, "Multi-fidelity Design Optimization of Lowboom Supersonic Business Jets". AIAA Paper 2004-1530.

[6] H. Hatanaka, S. Obayashi, S. Jeong. "Application of the Variable-fidelity MDO Tools to a Jet Aircraft Design". The 25th International Congress of the Aeronautical Science 2006.

[7] S.E. Gano, J.E. Renaud, J.D. Martin, T.W. Simpson. "Update Strategies for Kriging Models for Using in Variable Fidelity Optimization". AIAA Paper 2005-2057, April, 2005.

[8] C.Y. Tang, K. Gee, S.L. Lawrence. "Generation of Aerodynamic Data using a Design of Experiment and Data Fusion Approach". AIAA Paper 2005-1137, Jan, 2005.

[9] J. Sacks, W.J. Welch, T.J. Mitchell, H.P. Wynn. "Design and Analysis of Computer Experiments". Statistical Science, Volume 4, 1989, pp. 409-423.

[10] D. Schwamborn, T. Gerhold, R. Heinrich. "The DLR TAU-Code: Recent Applications in Research and Industry". ECCOMAS CDF 2006, The Netherland, 2006.

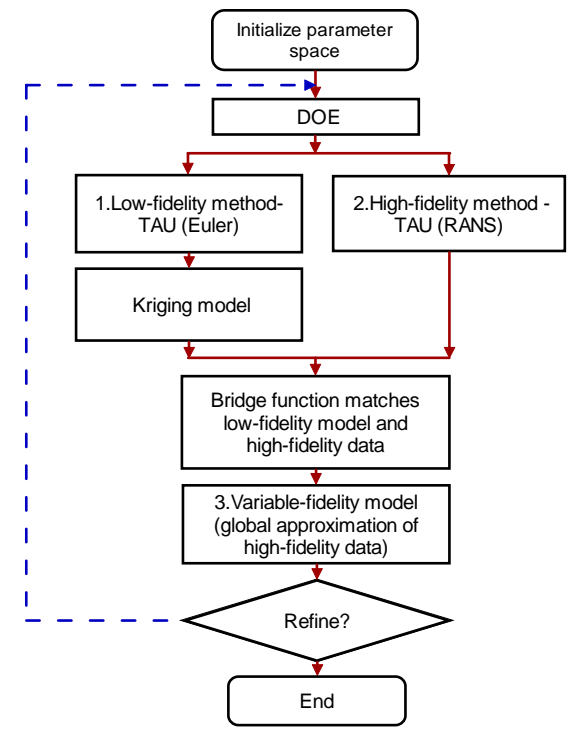

Figure 1 Framework of VFM for aero-loads prediction 


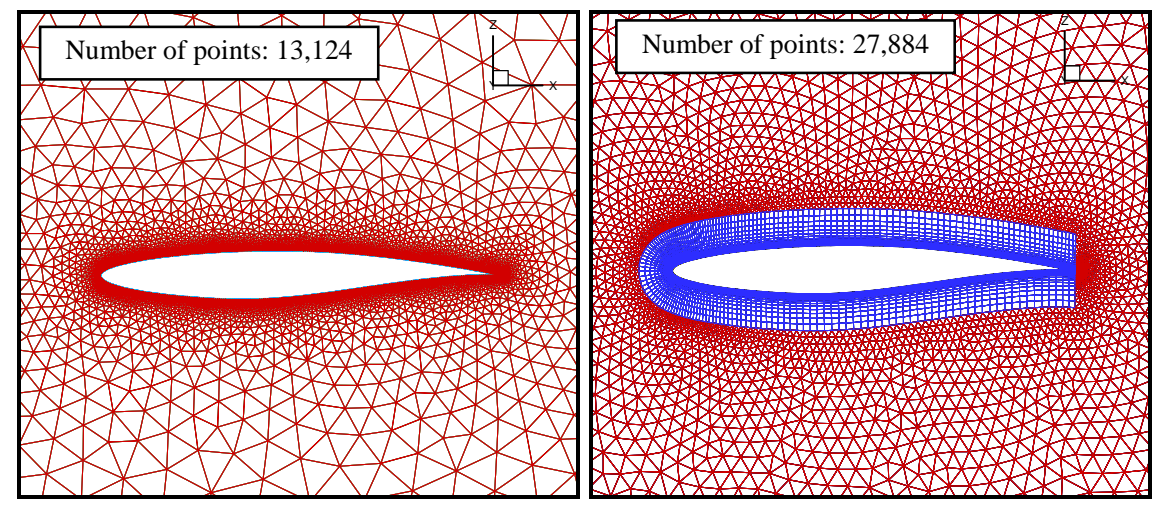

Figure 2 Computational grids for Euler and N-S calculation

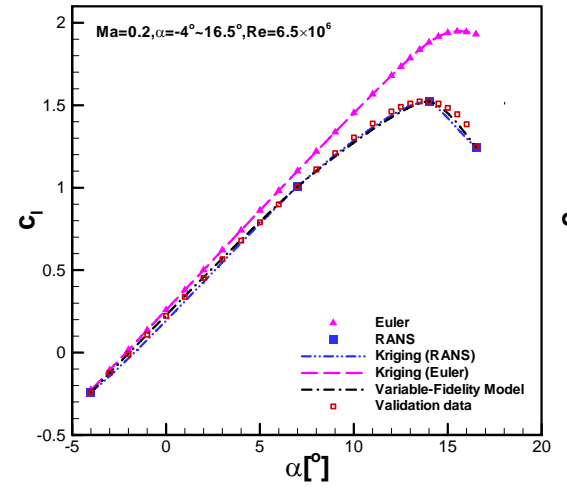

a. Lift

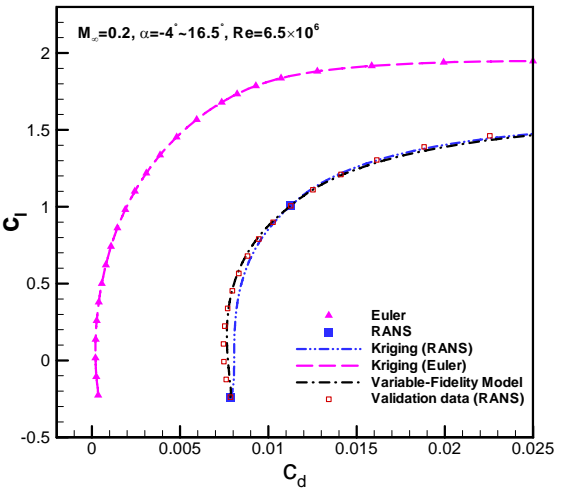

b. Drag

Figure 3 VFM for lift and drag polar of RAE 2822 airfoil ( $M a=0.2, \alpha=-4^{\circ} \sim 16.5^{\circ}$ )

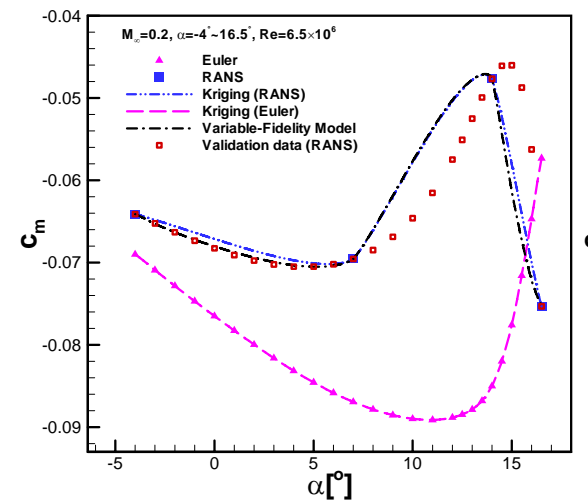

a. Before refinement

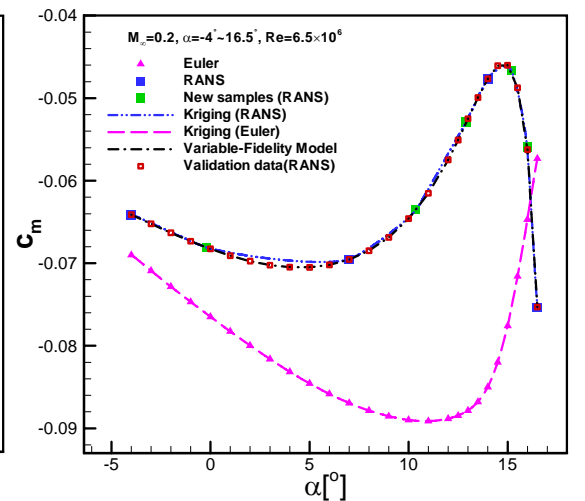

b. After refinement

Figure 4 VFM for $C_{m}$ of RAE 2822 airfoil $\left(M a=0.2, \alpha=-4^{\circ} \sim 16.5^{\circ}\right)$ 


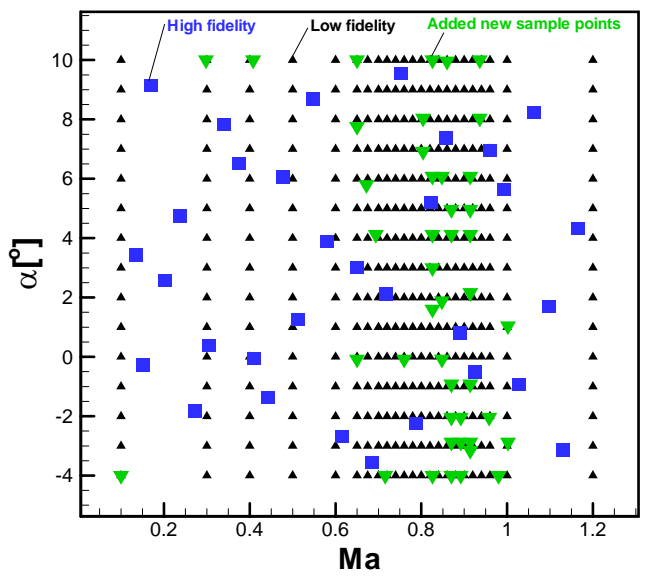

Figure 5 Samples used to construct VFM $\left(M a=0.1 \sim 1.2, \alpha=-4^{o} \sim 10^{\circ}\right)$

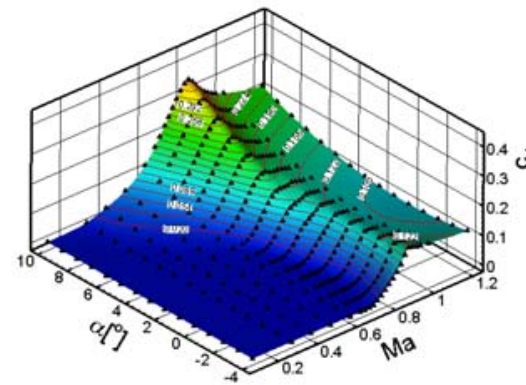

a. Low fidelity model (Euler, 345 samples)
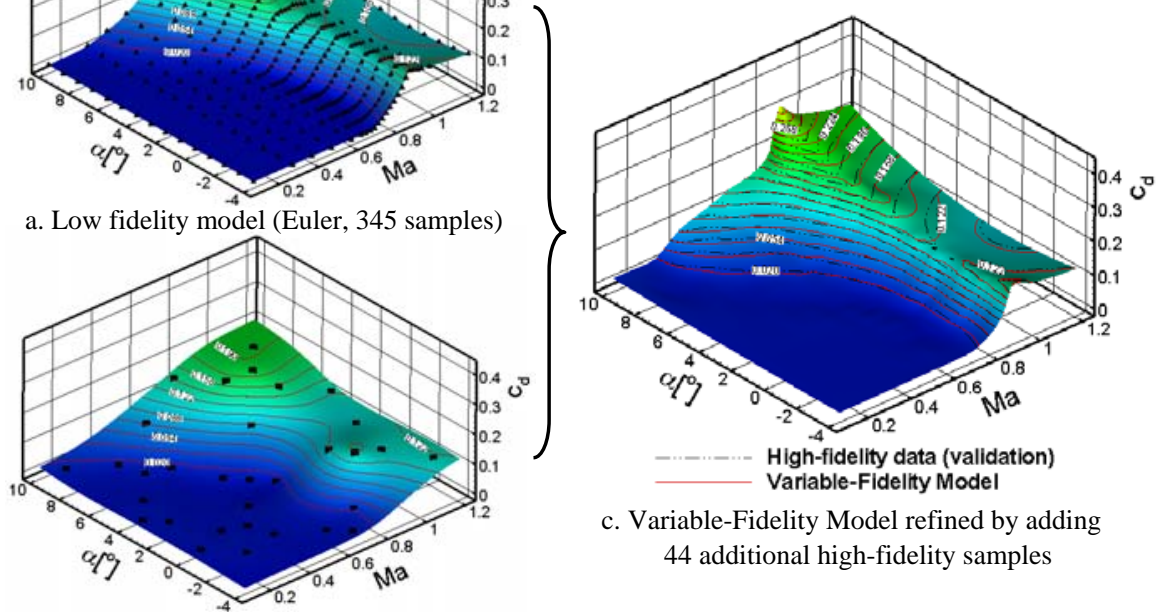

c. Variable-Fidelity Model refined by adding 44 additional high-fidelity samples

b. High fidelity model (RANS, 32 initial samples)

Figure 6 Variable-fidelity model for $C_{d}$ of RAE 2822 airfoil $\left(M a=0.1 \sim 1.2, \alpha=-4^{\circ} \sim 10^{\circ}\right.$ ) 